\title{
Preoperative MRI and immunohistochemical examination for the prediction of high-risk endometrial cancer
}

\author{
Jingya Chen ${ }^{1}$, Weimin Fan ${ }^{2}$, Hailei Gu ${ }^{3}$, Wei Zhang ${ }^{4}$, Yuting Liu ${ }^{5}$, Yajing Wang ${ }^{1}$, Zhaochun Pan ${ }^{1}$, \\ Zhongqiu Wang'
}

${ }^{1}$ Department of Radiology, Affiliated Hospital of Nanjing University of Chinese Medicine, Nanjing, China; ${ }^{2}$ Department of Clinical Laboratory, Women's Hospital of Nanjing Medical University (Nanjing Maternity and Child Health Care Hospital), Nanjing, China; ${ }^{3}$ Department of Radiology, Women's Hospital of Nanjing Medical University (Nanjing Maternity and Child Health Care Hospital), Nanjing, China; ${ }^{4}$ Department of Radiology, Affiliated Hospital of Integrated Traditional Chinese and Western Medicine, Nanjing University of Chinese Medicine, Nanjing, China; ${ }^{5}$ Department of Radiology, Children's Hospital of Nanjing Medical University, Nanjing, China

Contributions: (I) Conception and design: Z Wang, Z Pan; (II) Administrative support: Z Wang; (III) Provision of study materials or patients: W Fan, H Gu, W Zhang, Y Liu, YJ Wang; (IV) Collection and assembly of data: J Chen, Y Liu, Y Wang; (V) Data analysis and interpretation: J Chen, W Fan; (VI) Manuscript writing: All authors; (VII) Final approval of manuscript: All authors.

Correspondence to: Zhongqiu Wang; Zhaochun Pan. Department of Radiology, Affiliated Hospital of Nanjing University of Chinese Medicine, Nanjing, China. Email: zhqwang001@126.com; zhouping1014@126.com.

Background Magnetic resonance imaging (MRI) and immunohistochemical (IHC) examination provides useful information for the risk stratification of endometrial cancer (EC). However, the use of the combination of MRI and IHC for the prediction of high-risk EC is controversial. The aim of this study was to evaluate the value of preoperative MRI and IHC examination in prediction of patients with high-risk EC.

Methods: This retrospective case-control study was conducted from January 1, 2018 to May 1, 2021 at two hospitals. A primary cohort $(n=102)$ comprised patients with histologically confirmed EC in one hospital between January 1, 2018 and May 31, 2020. An additional external cohort ( $\mathrm{n}=35)$ comprising patients with histologically confirmed EC in a different hospital from January 1, 2020 to May 1, 2021 was included for validation. Imaging features including tumor size, tumor margin, relative $\mathrm{T} 2$ value, tumor signal intensity on diffusion-weighted imaging (DWI), T1-weighted imaging (T1WI), T2-weighted imaging (T2WI) were determined from preoperative MRI images. IHC markers including ER, PR, p53 and Ki67 were determined through IHC staining of preoperative curettage specimen. Patients were divided into high-risk and low-intermediate- risk group based on the final histological results. Differences between categorical and numerical variables were assessed using chi-square test and independent-sample t-test, respectively. Multivariate binary logistic regression analyses were used for construction of the prediction model A fusion prediction model was constructed by combining MRI features and IHC markers. The predictive performance of the model was then validated using the external cohort.

Results: Imaging and IHC markers were significantly associated with risk ranks. Model 1 based on MRI features showed an area under the curve (AUC) of 0.822 [95\% confidence interval (CI), 0.741-0.903] whereas Model 2 based on IHC markers showed an AUC of 0.894 (95\% CI, 0.829-0.960). Notably, model 3 integrating independent MRI and IHC risk factors demonstrated good calibration and high differentiation ability with an AUC of 0.958 (95\% CI, 0.923-0.993), and showed good discrimination with an AUC of 0.84 (95\% CI, 0.677-0.942) using the external validation set.

Conclusions: This study proposes a comprehensive predictive model comprising MRI and IHC features as a powerful tool for preoperative risk stratification to assist in clinical decision-making for EC patients.

Keywords: Endometrial cancer (EC); magnetic resonance imaging (MRI); immunohistochemistry; risk; prediction model 
Submitted Jan 18, 2021. Accepted for publication May 21, 2021.

doi: $10.21037 / g s-21-38$

View this article at: https://dx.doi.org/10.21037/gs-21-38

\section{Introduction}

Incidence of endometrial cancer (EC) has been increasing exponentially in recent years worldwide (1). Most ECs can be diagnosed in the early stage due to their apparent clinical symptoms, therefore, patients have a better prognosis. However, some poorly differentiated and atypical histological types of EC may present with lymphatic vascular invasion (LVSI) or distant metastasis even in the early stage resulting in a poor outcome. Therefore, NCCN guidelines (2) stratify patients with EC into high-, intermediate- and low-risk subgroups which is useful in guiding adjuvant therapy. Currently several guidelines have been published for clinical practice (2-4), however, the recommended surgical strategies and adjuvant therapy for patients in different risk groups are significantly different. Therefore, it is important to accurately classify patients of high-risk EC group before surgery thus providing appropriate treatment plans. Recent studies used preoperative imaging examinations and curettage pathology to distinguish between different risk groups. Liu et al. (5) reported that ADC has the potential to discriminate intermediate- and high-risk stage I EC patients, whereas Lee et al. (6) reported that preoperative MRI and histological results can be used for identification of low-risk EC patients. However, results of risk prediction of EC using MRI or IHC alone show significant variations (6-9). Therefore, it is important to explore the effect of combining preoperative MRI features and IHC markers on the predictive value for high-risk EC. This study sought to establish a predictive model by combining preoperative MRI features and IHC markers which are easily accessible in clinical practice. The effectiveness of the model was externally validated and compared with efficacy of predictive models based on MRI or IHC separately.

We present the following article in accordance with the TRIPOD reporting checklist (available at https://dx.doi. org/10.21037/gs-21-38).

\section{Methods}

\section{Study population}

The study was approved by the institutional review board of the Affiliated Hospital of the Nanjing University of Chinese Medicine (No. 2016NL-005-02). The study was conducted in accordance with the Declaration of Helsinki (as revised in 2013). Informed consent was waived as this is a retrospective case-control study. Sample size was calculated based on the area under the ROC curve (AUC). The minimum AUC for the prediction of high-risk group EC was expected to be 0.70 based on null hypothesis of 0.50 . The minimum acceptable sample size was 41 participants for each group using a $5 \% \alpha$ risk and $10 \% \beta$ risk. Statistical analyses were performed using MedCalc (V 19.4.0, Belgium). A total of 192 patients with histologically confirmed EC in our center between January 1, 2018 and May 31, 2020, and 64 patients with histologically confirmed EC in Women's Hospital of Nanjing medical University from January 1, 2020 to May 1, 2021 were enrolled in this study. Notably, 119 patients were excluded based on the following criteria: (I) MRI scanning was not performed ( $\mathrm{n}=11)$; (II) tumor lesions were not visible on MRI images ( $\mathrm{n}=32$ ); (III) MRI examinations were conducted more than 2 weeks before curettage or surgery $(n=22)$; (IV) poor quality of MRI images for evaluation $(n=23)$ and $(V)$ preoperative curettage was not performed $(n=31)$. Ultimately, 102 patients were included in the primary cohort, and 35 patients were included in the validation cohort. Participant selection details are presented in the flow chart shown in Figure 1. All enrolled patients underwent radical hysterectomy. Final pathological assessments were performed using surgical specimens.

Patients were divided into two groups based on the final histopathological diagnosis of the subtype and grade of the tumor for analysis. The groups included lowintermediate-risk (LI-risk) group and high-risk group. The low-intermediate-risk group comprised patients with grade 1 and grade 2 endometrioid cancer with deep myometrial invasion (DMI) $<50 \%$, whereas the high-risk group comprised patients with non-endometrioid tumors or grade 3 endometrioid cancer with $\mathrm{DMI} \geq 50 \%$ regardless of histopathology subtypes (10). Clinical and pathological characteristics of patients are presented in Table 1.

\section{MRI protocol and Image analysis}

MRI examination was performed using a $1.5 \mathrm{~T}$ MRI 

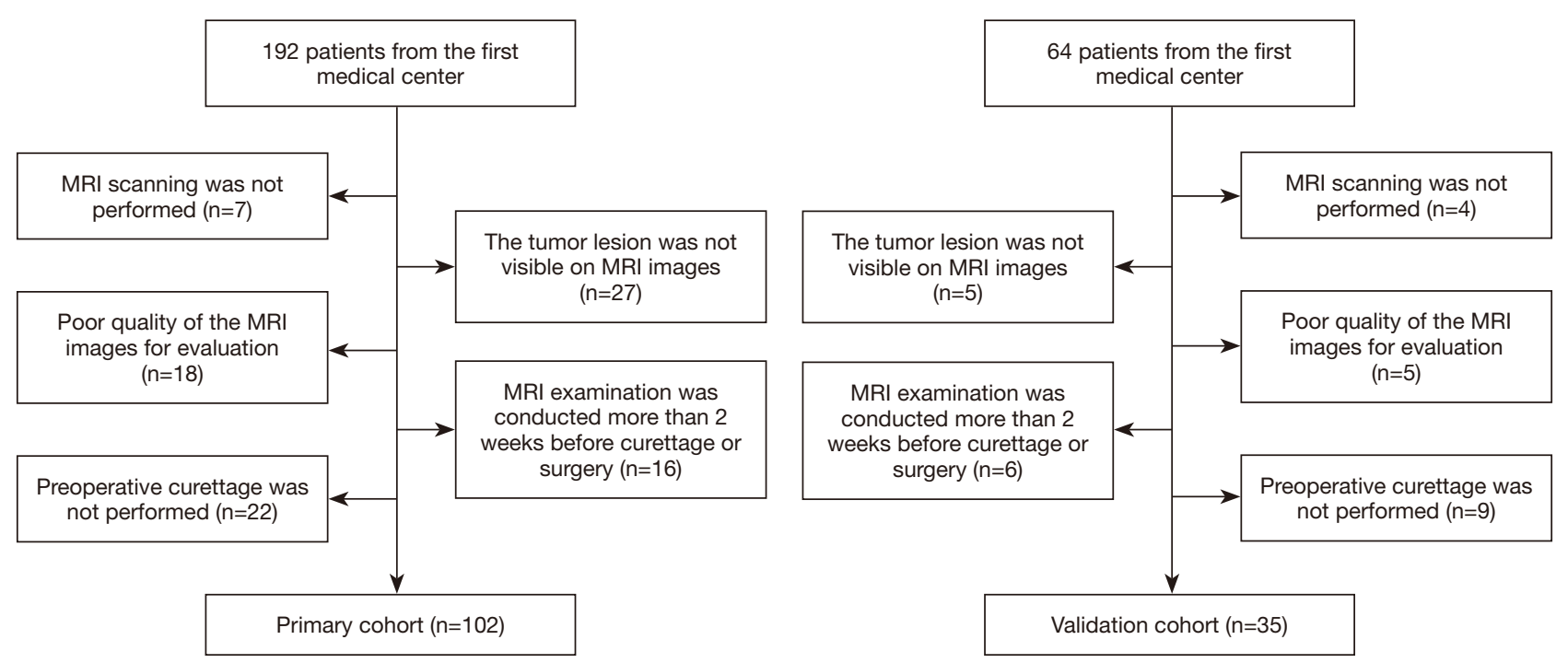

Figure 1 Flowchart of inclusion process of the study.

equipment (Magnetom Aera; Siemens Healthcare, Erlangen, Germany) covering the whole pelvic area. Axial T1WI [time of reception (TR)/time of echo (TE), $145 \mathrm{~ms} / 4.6 \mathrm{~ms}$ ] and axial T2WI (TR/TE, 1,800 ms/75 ms) with an interaction gap of $1 \mathrm{~mm}$ and a thickness of $6.0 \mathrm{~mm}$, and sagittal fatsuppress (FS)-T2WI (TR/TE, $260 \mathrm{~ms} / 4.6 \mathrm{~ms})$ with an interaction gap of $1 \mathrm{~mm}$ and a thickness of $5.0 \mathrm{~mm}$ were evaluated. Moreover, sagittal DWI (TR/TE, $6,900 \mathrm{~ms} / 80 \mathrm{~ms}$ ) with an interaction gap of $1 \mathrm{~mm}$, a thickness of $6.0 \mathrm{~mm}$, and a b-value of 50 and $800 \mathrm{~s} / \mathrm{mm}^{2}$ was determined. ADC maps were then generated using an automatic post process.

Two board-certified radiologists with 5 and 12 years of experience in abdominal MRI diagnosis examined the images independently. In case of discordance, a consensus was reached through discussion. Tumor size, tumor margin (well-defined or ill-defined), and relative $\mathrm{T} 2$ value were measured. In addition, tumor signal intensity on DWI, T1WI (hyper- or iso-intensity), and T2WI (hetero- or homogeneous) were recorded. DMI was defined as the deepest measurement assessed on T1WI, T2WI and DWI images. The regions of interest (ROI) were identified manually on the slice of the tumor which exhibited the largest diameter whereas cystic areas were avoided.

\section{Tissue and immunohistochemistry (IHC) analysis}

All histopathological slides were reviewed by a pathologist with 8 years of experience in gynecological malignancies who had no prior knowledge of patient information. Tissue sections were fixed in formalin for 8-24 hours and then embedded in paraffin for $\mathrm{H} \& \mathrm{E}$ staining. After examining expression of ER (DAKO, 1D5, Denmark), PR (DAKO, PgR636, Denmark), and ki-67 (DAKO, MIB-1, Denmark), the percentage of positive tumor cells was determined. In addition, P53 (Maixin, MX008, China) was classified as either wild type or mutant type. Clinicopathological features including stage, subtype, grade, DMI, and lymphovascular invasion (LVI) were recorded.

\section{Statistical analysis}

Complete cases were included for statistical analysis. All data analyses were performed using IBM SPSS (Version 21.0; IBM Corp., NY, USA). Intraclass correlation coefficient (ICC) or Kappa coefficient were computed to determine the interrater reliability. For quantitative variables, data normality tests were performed using Shapiro-Wilk test and presented as mean \pm SD. Group comparison was performed using student $t$-test. Qualitative variables were expressed as percentages and compared using chi-square test or Fisher's exact test.

\section{Prediction model construction and validation}

Multivariate logistic regression analyses were used to develop prediction models for differentiation between the two risk groups. The predictive values of the regression models were assessed using receiver operating characteristic 
Table 1 Characteristics of patients in the primary and validation cohorts

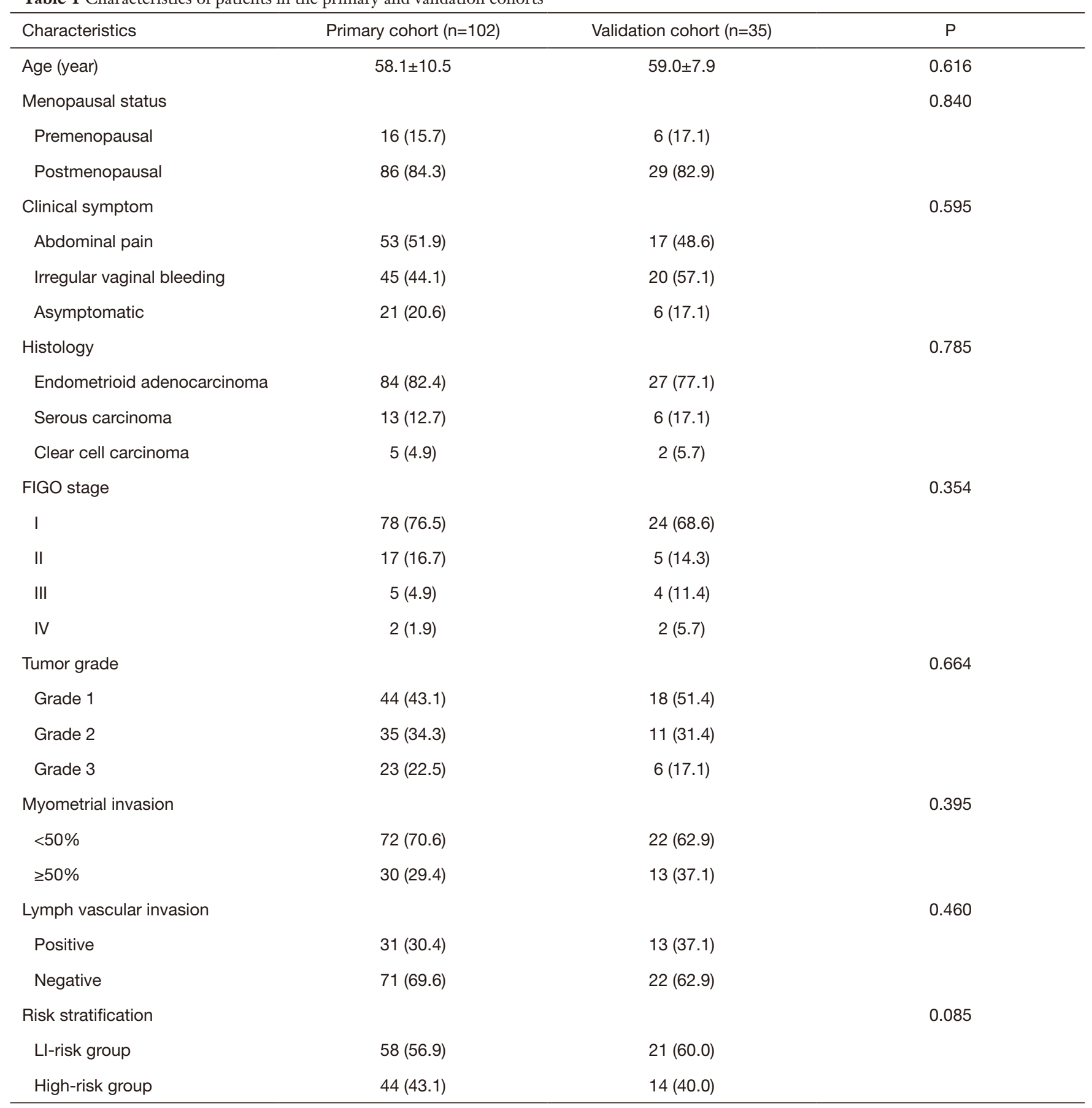

Values are given as $\mathrm{n}(\%)$ or mean $\pm \mathrm{SD}$. LI-risk, low-intermediate-risk.

(ROC) curve analysis and area under the curve (AUC). DeLong's test was used to evaluate the clinical efficacy by comparing the AUC of each predictive model. HosmerLemeshow test was performed to determine the calibration capability of prediction models. Generalizability of prediction model was verified with the external validation cohort. Significance level was set at $\mathrm{P}<0.05$ for all statistical tests. 


\section{Results}

\section{Clinical and pathological characteristics}

A total of 102 patients (average age of $58.1 \pm 10.5$ years) were included in the primary cohort. High-risk group rate among the 102 patients was $43.1 \%(n=44)$. Thirty-five patients (average age of $59.0 \pm 7.9$ years) were included in the validation cohort. High-risk group rate of the validation cohort was $40 \%(n=14)$. Characteristics of patients in the primary and validation cohorts are presented in Table 1 . Analysis showed no statistically significant differences in age, menopausal status, clinical symptom, tumor histology, FIGO stage, tumor grade, myometrial invasion, lymphovascular invasion and risk stratification between the two cohorts $(\mathrm{P}=0.616,0.840,0.595,0.785,0.354,0.664$, $0.359,0.460$ and 0.085 , respectively).

\section{Immunobistochemical and MRI characteristics}

Expression levels of ER, PR, Ki-67, and p53 were determined through immunohistochemical analysis from the samples obtained using diagnostic curettage and definitive operation. Analysis of IHC markers of the preoperative procedure and the definitive histopathological results showed statistically significant correlation (all $\mathrm{P}<0.05$ ). Notably, LI-risk group showed a significantly higher expression of ER and PR compared with the level in high-risk EC patients $(\mathrm{P}<0.05)$. In addition, patients with high-risk EC showed higher levels of ki67 $(\mathrm{P}<0.01)$ compared with those with LI-risk EC. Mutated p53 was highly abundant in the high-risk group compared with LI-risk EC group (Table 2).

Analysis of tumor size, tumor margin, relative T2 value, DMI status, tumor signal intensity on DWI, T1WI, and T2WI showed good overall interrater reliability (ICC $=0.834,0.762,0.804,0.698,0.882,0.965$, and 0.702 , respectively). Analysis of age, menopausal status, tumor size, tumor margin, the signal on DWI, T1WI, or T2WI in the primary cohort showed no significant differences between the two risk groups. Analysis of age, menopausal status, $\mathrm{PR}$, tumor size, tumor margin, the signal on DWI, T1WI, or T2WI in the validation cohort showed no significant differences observed between the two groups. Notably, tumors of high-risk patients in both cohorts indicated lower $A D C$ values ( $\mathrm{P}=0.003$ and $\mathrm{P}<0.01$, respectively) and larger size $(\mathrm{P}<0.01$ and $\mathrm{P}=0.02$, respectively) compared with those of the LI-risk patients. See Figures 2 and 3 for examples. In addition, the high-risk group showed a higher rate of DMI in tumors compared with the low-risk group (Table 2).

\section{Performance of prediction models}

Multivariate logistic regression analyses were performed based on the variables which were significantly different between the two risk groups to determine the independent predictors and construct prediction models for discrimination of high-risk from LI-risk EC groups (Table 3). A score for each risk factor of the patient was calculated using the models, and the total risk score was obtained by adding the scores for the risk factors. Model 1 was based on preoperative MRI measurements (tumor size, ADC value, relative-T2 value, and DMI status). Analysis showed that $\mathrm{ADC}$ value $(\mathrm{OR}=1.956 ; 95 \% \mathrm{CI}, 1.360-2.812)$ and DMI status (OR $=0.996$; 95\% CI, 0.993-0.999) were independent markers for differentiation. Model 2 was established using IHC markers (ER, PR, ki67, and p53) of preoperative curettage tissue. Analysis showed that ER (OR $=0.961 ; 95 \%$ CI, 0.941-0.983), PR (OR =0.975; 95\% CI, 0.958-0.993) and Ki67 (OR =1.956; 95\% CI, 1.360-2.812) were independent markers of differentiation between LI-risk and high-risk EC groups. Further, model 3 was constructed based on combination of all MRI and IHC variables. Analysis of model 3 showed that ER (OR $=0.852 ; 95 \%$ CI, 0.627-0.978), Ki67 (OR =1.756; 95\% CI, 1.022-2.091), DMI (OR =2.224; 95\% CI, 1.458-3.636) and $\mathrm{ADC}$ value $(\mathrm{OR}=0.793 ; 95 \% \mathrm{CI}, 0.689-0.997$ ) were independent markers in this model. AUC of the 3 models (model 1, 2 and 3) were 0.822 (95\% CI, 0.741-0.903), 0.894 (95\% CI, 0.829-0.960), and 0.958 (95\% CI, $0.923-$ $0.993)$, respectively (Table 3). Sensitivity and specificity of the 3 models were $(75 \%, 95 \%),(75 \%, 79 \%)$ and $(89 \%$, $93 \%$ ), respectively (Table 4 ). There were significant differences between these three ROC curves (Table 4). The results of Hosmer-Lemeshow test for the three models were $\chi^{2}=5.635(\mathrm{P}=0.691), 10.393(\mathrm{P}=0.211)$, and 7.237 $(\mathrm{P}=0.511)$, respectively. External validation showed good discrimination with an AUC of 0.84 (95\% CI, 0.677-0.942), with a sensitivity of $78.57 \%$, a specificity of $76.19 \%$. ROC curves and AUCs of predicting models in high-risk EC prediction are presented in Figure 4.

\section{Discussion}

In this study, predictive model 1 based on MRI features performed well for differential diagnosis between the two risk groups of ECs preoperatively, with an AUC of 0.822 , sensitivity of $75 \%$ and specificity of $90 \%$. Predictive performance of model 2 which was based on IHC markers 
Table 2 Comparisons of clinical, pathological and imaging features between LI-risk and high-risk group of endometrial cancer patients

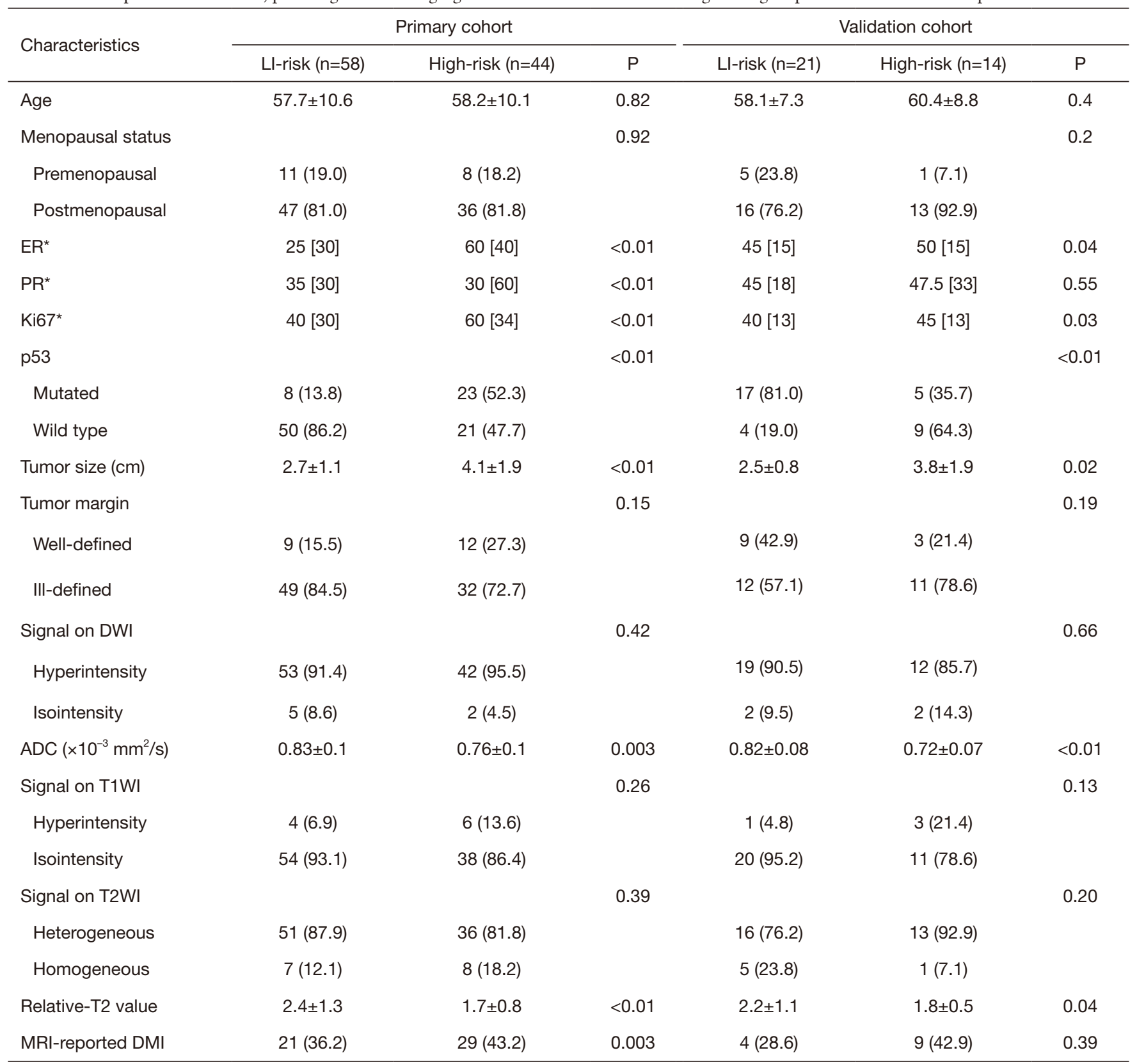

Values are given as $\mathrm{n}(\%)$, mean $\pm \mathrm{SD}$ (range), or ${ }^{*}$ median [IQR]. IQR, interquartile range; ER, estrogen receptor; PR, progesterone receptor; ADC, apparent diffusion coefficient; T1WI, T1-weighted imaging; T2WI, T2-weighted imaging; MRI, magnetic resonance imaging; $\mathrm{DMI}$, deep myometrial invasion; LI-risk, low-intermediate-risk.

of preoperative curettage specimens performed better compared with model 1, with an AUC of 0.894 , sensitivity of $83 \%$ and specificity of $96 \%$. To explore the synergistic prediction effect of these two preoperative examinations models, model 3 was developed using preoperative MRI and IHC variables. The AUC of model 3 was 0.958 , sensitivity was $89 \%$ and specificity was $93 \%$, implying that preoperative imaging and IHC examination are important in the preoperative risk assessment of EC. Notably, the combined model from these two techniques showed a higher differential value compared with the individual techniques. Therefore, model 3 consisting of MRI and IHC features can 

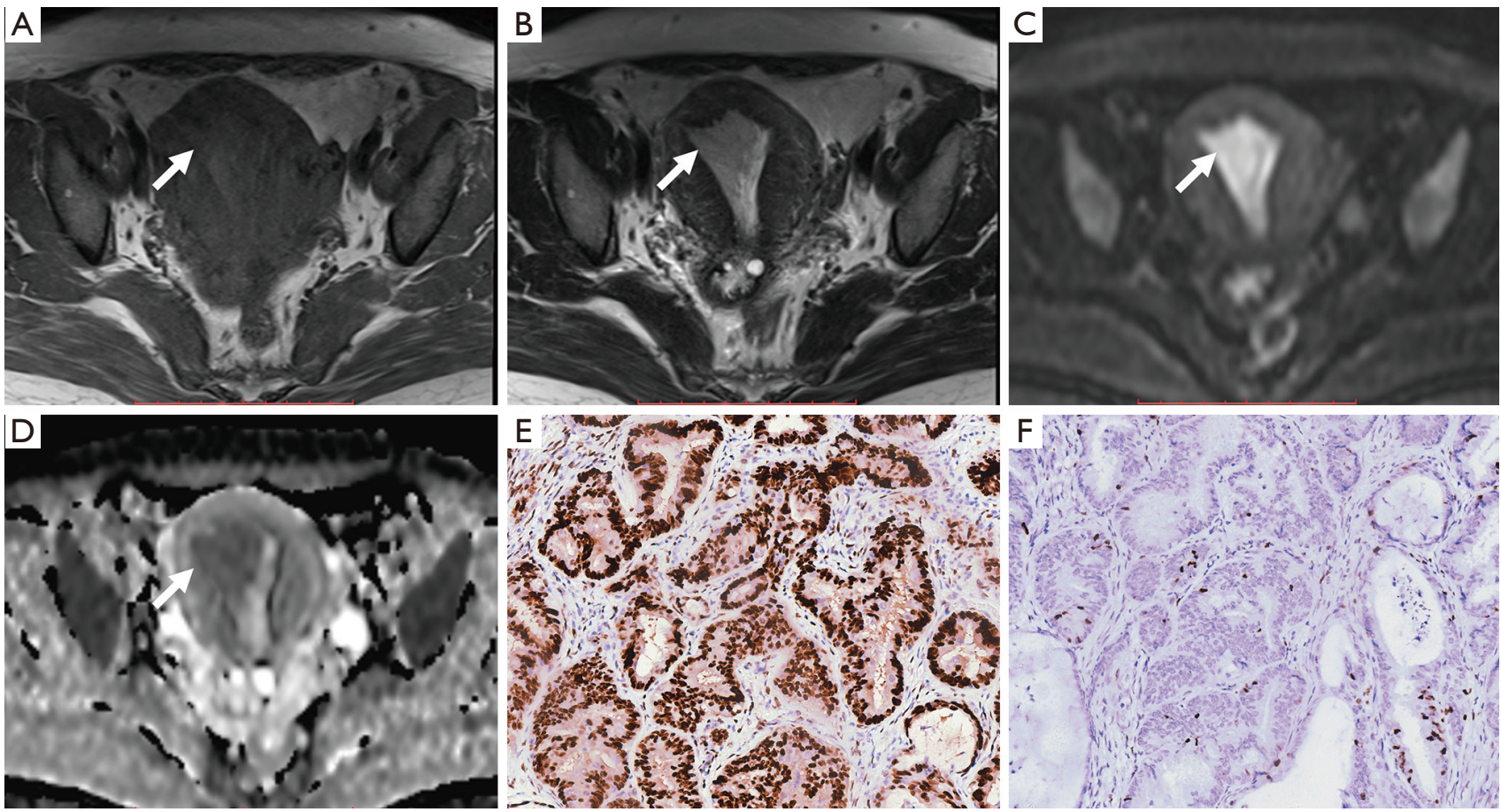

Figure 2 Representative grade 1 EC images from a 43-year-old patient. (A) Transverse plane T1W, and (B) T2W MR image shows an intracavitary lesion (white arrows in A, B, C and D) with isointensity and hyperintensity, respectively. The tumor demonstrates significantly high signal intensity on DWI $(\mathrm{b}=800)(\mathrm{C})$ and moderate low signal intensity ( $\mathrm{ADC}=0.793 \times 10^{-3} \mathrm{~mm}^{2} / \mathrm{s}$ ) on ADC maps (D). (E) Preoperative IHC results showed that the expression of ER in tumor tissues was diffuse and strongly positive, approximately $90 \%$ positive (original magnifications $\times 200$ ). (F) Expression of Ki-67 in tumor tissues was focally positive, approximately $5 \%$ positive (original magnifications $\times 200)$. EC, endometrial cancer; DWI, diffusion-weighted imaging; ADC, apparent diffusion coefficient; ER, estrogen receptor.

be used for preoperative risk stratification of EC.

The predictive performance of model 1 based on preoperative MRI features was moderate ( $\mathrm{AUC}=0.822$, sensitivity $=75 \%$, a specificity of $90 \%$ ), with high specificity implying that it can be used to identify LI-risk EC patients accurately. Several studies report that MRI parameters can be used for preoperative assessment of EC $(5,11,12)$. In addition, Nougaret et al. (11) explored the correlation between ADC, tumor grade, and LVSI and reported that ADC values of G3 EC were significantly lower compared with those of G1/G2 EC. A study by Zhang et al. (13) reported that the area under the ROC curve (AUC) of ADC values was 0.892 in the identification of the low-risk EC. Moreover, Shen et al. (14) assessed the predictive value of ADC in low-risk EC subjects. In summary, these studies show that $\mathrm{ADC}$ is higher in low-risk EC patients compared with high-risk EC patients. The highest value of AUC for low-risk EC patients was 0.876 (95\% CI, 0.798-0.954). In the present study, $\mathrm{ADC}$ value was identified as a significant predictor of tumor risk stratification in EC with an OR of 1.956 (95\% CI, 1.360-2.812), which was consistent with previous results. Therefore, these findings show that preoperative tumor $\mathrm{ADC}$ values can be used to assess the risk level of EC particularly for patients with low-risk EC. Moreover, DMI $\geq 50 \%$ is an independent prognostic factor for EC (15). In addition, European Society for Medical Oncology (ESMO) reported that DMI can be used as a criterion for classification of patients of different risk groups. Dane et al. (16) explored the relationship between myometrial invasion and progression-free survival (PFS) or overall survival (OS) in EC. Their findings showed that patients with DMI exhibited a shorter PFS and OS, implying that DMI is an important prognostic indicator and can be used as a determinant of treatment in EC. In the current study, ADC value and DMI were significant predictors for differentiating high-risk from LI-risk EC, which was inconsistent with previous reports. In addition, studies report that tumor size plays an important role 

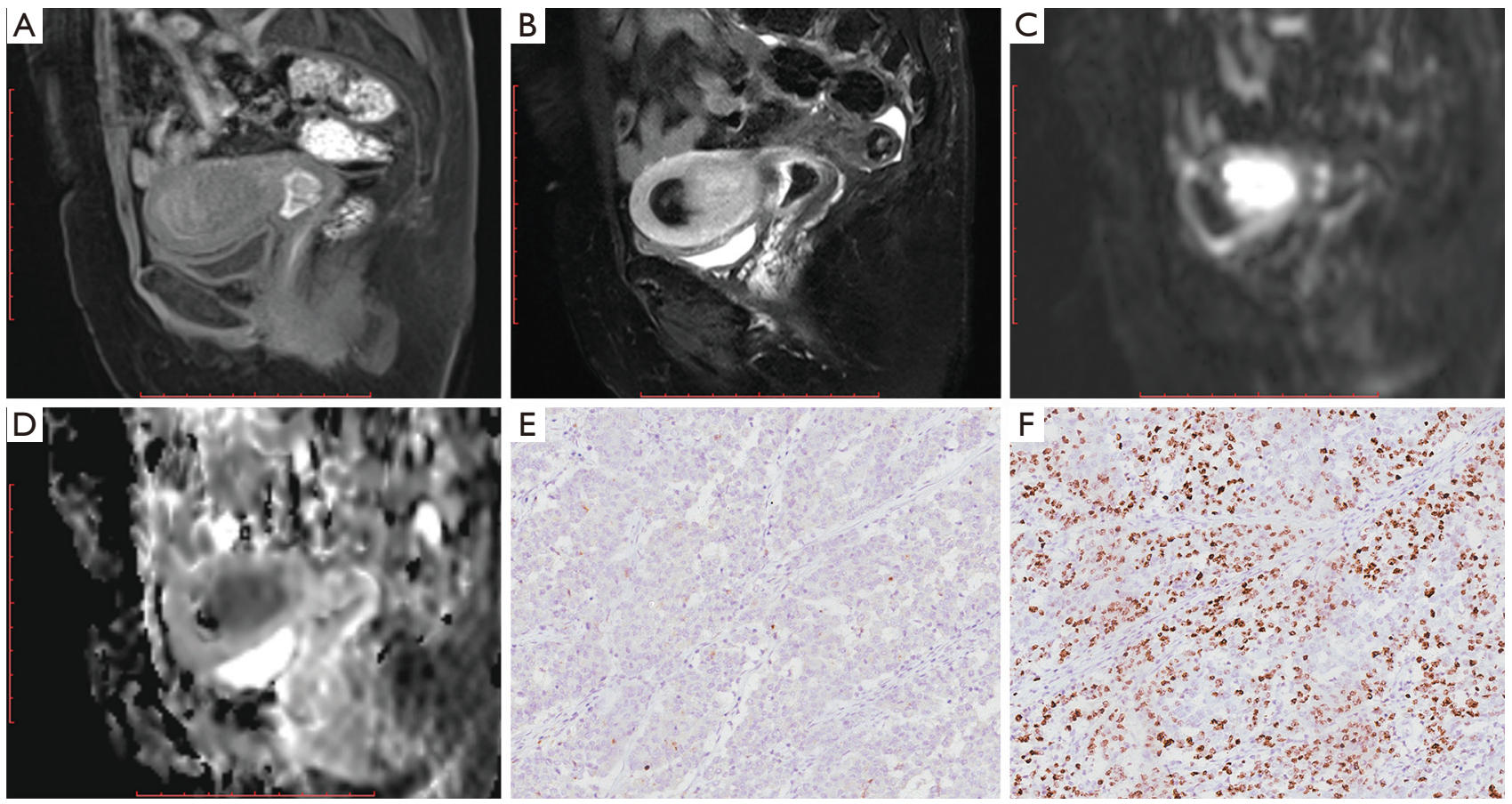

Figure 3 Images of a 63-year-old woman with histopathologically diagnosed grade 3 endometrioid carcinoma. (A) Sagittal plane T1W MR image showing a large isointense lesion filling the endometrial cavity. (B) On T2W imaging the tumor shows slight hyperintensity compared with adjacent myometrium. (C) On DWI ( $b=800)$, the tumor shows significant hyperintensity compared with adjacent myometrium, whereas (D) on the ADC map it appears as dark signal areas $\left(\mathrm{ADC}=0.65 \times 10^{-3} \mathrm{~mm}^{2} / \mathrm{s}\right.$ ), which represent water restriction. Preoperative $\mathrm{IHC}$ results, (E) expression of ER in tumor tissues was negative (original magnifications $\times 200$ ), whereas (F) expression of Ki-67 in tumor tissues was diffuse positive, approximately $60 \%$ positive (original magnifications $\times 200$ ). DWI, diffusion-weighted imaging; ADC, apparent diffusion coefficient.

Table 3 Multivariate logistic regression models for differentiation of low-risk and high-risk endometrial cancer

\begin{tabular}{|c|c|c|c|c|}
\hline Model & Variables & Regression coefficient & OR $(95 \% \mathrm{Cl})$ & $P$ value \\
\hline Model 1 & DMI & -0.04 & $0.996(0.993-0.999)$ & 0.005 \\
\hline \multirow[t]{2}{*}{ Model 2} & Ki67 & 0.051 & $1.053(1.023-1.083)$ & 0.001 \\
\hline & ER & -0.039 & $0.961(0.941-0.983)$ & 0.001 \\
\hline \multirow[t]{4}{*}{ Model 3} & Ki67 & 0.054 & $1.756(1.022-2.091)$ & 0.003 \\
\hline & ER & -0.49 & $0.852(0.627-0.978)$ & 0.005 \\
\hline & DMI & -0.965 & $2.224(1.485-3.636)$ & 0.021 \\
\hline & ADC & 0.007 & $0.793(0.689-0.997)$ & 0.015 \\
\hline
\end{tabular}

OR, odds ratio; ER, estrogen receptor; PR, progesterone receptor; ADC, apparent diffusion coefficient; DMI, deep myometrial invasion. 
Table 4 The statistical difference between three ROC curves

\begin{tabular}{lccccc}
\hline Model & AUC $(95 \%$ Cl) & SEN $(\%)$ & SPE (\%) & Z & P value \\
\hline Model 1 & $0.822(0.741-0.903)$ & 75 & 97 & $1.443^{\mathrm{a}}$ & $0.0149^{\mathrm{a}}$ \\
Model 2 & $0.894(0.829-0.960)$ & 75 & 79 & $2.429^{\mathrm{b}}$ & $0.0152^{\mathrm{b}}$ \\
Model 3 & $0.958(0.923-0.993)$ & 89 & 93 & $3.577^{\mathrm{c}}$ & $0.0003^{\mathrm{c}}$ \\
\hline
\end{tabular}

${ }^{a}$, Model 1 vs. Model 2; ${ }^{\text {, }}$, Model 3 vs. Model 1; ${ }^{~,}$, Model 3 vs. Model 2. SEN, sensitivity; SPE, specificity.
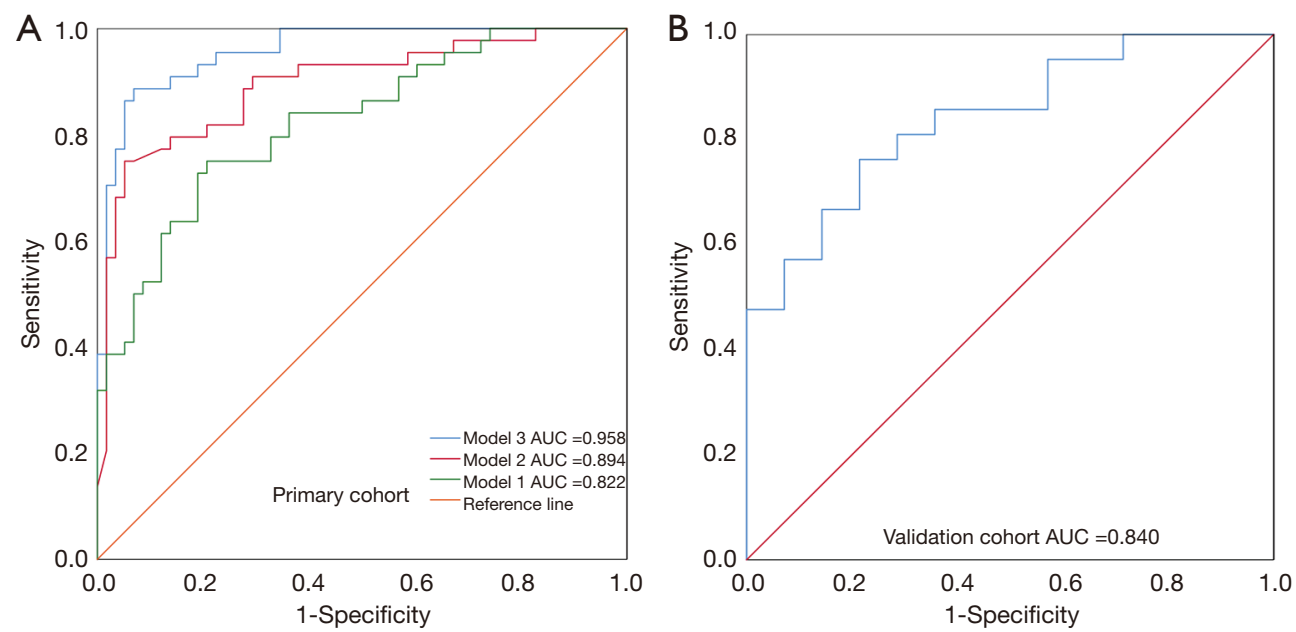

Figure 4 ROC curves of the models in primary and validation cohorts. (A) ROC curves of the performance of the model 1 , model 2 , and model 3 for high-risk EC prediction in primary cohort. AUC was 0.822 for model 1 (95\% CI, 0.692-0.882; $\mathrm{P}<0.05$ ), 0.894 for model 2 (95\% CI, 0.829-0.960; $\mathrm{P}<0.05$ ), and 0.985 for model 3 (95\% CI, 0.923-0.993; $\mathrm{P}<0.05$ ). (B) In the validation cohort, the AUC of model 3 was 0.840 (95\% CI, 0.677-0.942; $\mathrm{P}<0.05$ ). ROC, receiver operating characteristic; AUC, area under the curve; EC, endometrial cancer.

in preoperative evaluation and therapy planning $(3,12)$. However, in this study, this parameter was not statistically different in multivariate logistic regression analyses and thus was not selected for construction of model 2. This insignificance may be caused by specific criteria of different studies for the risk stratification of EC. Non-endometrioid type of EC was also included in the high-risk group in this study. This type of EC is characterized by a small size with more malignant characteristics, which may lead to inconsistency in results. Therefore, further studies using larger sample sizes and multimodal MRI parameters should be conducted to confirm these intriguing results.

Furthermore, the predictive performance of model 2 based on IHC markers of curettage tissue showed good performance in preoperative risk stratification of EC. Preoperative endometrial sampling is an important approach for clinically suspected endometrial lesions, however, the conventional pathological technique is insufficient for risk assessment of EC. Notably, IHC examination can significantly improve the accuracy of diagnosis (7). Several studies report that IHC markers are important for identification of different pathologic subtypes and risk factors of EC. A study by Mhawech-Fauceglia et al. (17) used ER, PR, insulin-like growth factor II mRNA-binding protein 3 (IMP3), and intestinal trefoil factor 3 (TFF3) to distinguish endometrioid carcinoma from serous and clear cell carcinoma. Analysis showed that combination of ER (+)/PR (+)/TFF3 (+)/IMP3 (-) immunologic features was effective in diagnosis of endometrioid carcinoma subtypes

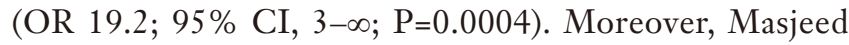
et al. (18) reported that the ER, PR, p53, Ki67, and IHC markers can effectively reveal benign and malignant biological characteristics of endometrial tumors. These outcomes were statistically correlated with tumor degree and recurrence of endometrial carcinoma. However, findings from previous findings were inconsistent with the findings from our study in that the findings from our study did not show sufficient evidence for risk stratification of EC using 
markers such as Ki-67, vimentin, and other IHCs. The IHC markers may be predictors of the histopathologic grade of tumors, however, more studies should be performed to validate the specific correlation between these factors and malignant degree of EC. Notably, Guan et al. (19) reported that ER/PR were independent predictors of PFS and OS. Patients with ER/PR loss exhibited deeper muscular infiltration, advanced FIGO staging, and a higher rate of pelvic lymph node metastasis, resulting in shorter PFS and OS. Furthermore, Di Donato et al. (20) reported that ER and PR loss were more mainly found in G3 tumors. High expression of Ki-67 is significantly associated with DMI, and these three markers (ER, PR and Ki67) are independent predictor factors for endometrial malignancy. In the current study, lower ER and PR expression, and higher Ki-67 were observed in FIGO grade 3 endometrioid and non-endometrioid carcinoma, which was consistent with previous reports. Han et al. (21) explored the differential diagnostic value of 12 IHC markers in highgrade endometrial carcinoma. Analysis showed that multiple-IHC marker panels significantly improved the diagnostic accuracy of EC subtypes. IHC markers in several previous studies were obtained from a definitive gross specimen, whereas in the current study they were obtained from preoperative curettage specimens. Subsequently, this was valuable to surgeons in making a viable decision before surgery. Model 2 is a three-IHC marker panel with good performance in risk stratification of EC. The markers used in model 2 are routinely used in clinics with no extra cost.

Imaging or histological information provided by MRI or endometrial biopsy is useful but insufficient for accurate preoperative assessment. Therefore, the predictive efficacy of model 3 was determined by combining model 1 and model 2. Clinical efficacy of model 3 was statistically better compared with that of model $1(\mathrm{Z}=2.429, \mathrm{P}=0.015)$ and model $2(Z=3.577, P=0.0003)$, respectively. Notably, the difference between model 1 and model 2 was not statically significant $(Z=1.443, P=0.149)$. These findings show that this model had the best AUC and highest sensitivity compared with other models, however the specificity was compromised. These outcomes imply that the combination of preoperative MRI and IHC results can accurately identify high-risk EC, thus promoting a precise treatment for these patients. A study by Luomaranta et al. (9) reported a PPV of $74.4 \%$ and NPV of $86.1 \%$ for the detection of highrisk EC using combination of preoperative histology and MRI. However, IHC examination was not assessed in their study. Furthermore, Weinberger et al. (22) reported that a new model incorporating clinical data and IHC markers increased the diagnostic accuracy of prognosis prediction with a sensitivity of $75.8 \%$. Lee et al. (6) identified lowrisk EC patients based on MRI and histological results and obtained an AUC of 0.70 (95\% CI: 0.65-0.75) for the diagnosis of low-risk patients. In summary, ADC value, DMI status, ER, and Ki67 are significant predictors for differentiating high-risk EC from LI-risk EC. The sensitivity and specificity of model 3 were 0.886 and 0.934 , respectively. Model 3 showed moderate discrimination ability in the external validation set. This performance may be partly attributed to the small sample number, therefore, further evaluation using larger sample size should be performed. These results were consistent with previous conclusions, that preoperative IHC examination are useful in assessing risk groups and thus can be used to optimize therapy. Notably, model 3 (comprising of ADC value, DMI status, ER, and Ki67) is highly valuable in clinical application.

Despite these intriguing results, this study had a few limitations. First, serum markers were not included for the evaluation. A recent study (23) report that CA-125 exhibits an imperative role in predictive models. Therefore, future studies should incorporate serum markers such as CA-125 or L1CAM for more reliable results. Second, parameters of dynamic contrast-enhanced (DCE) MRI were not included in this work due to high medical cost. Although the performance of model 3 was good, further studies comprising DCE-MRI sequences should be performed. Lastly, the sample size of the validation set was small, therefore the validated results were not reliable. Therefore, a multi-center program with larger sample sizes should be conducted for further validation of these results.

\section{Conclusions}

In summary, preoperative MRI and IHC parameters are valuable markers for risk stratification of EC. Model 3 comprising preoperative MRI and IHC exhibited a good discriminatory ability in high-risk EC discrimination.

\section{Acknowledgments}

The language of this paper is edited by professional editor from freescience company.

Funding: This study was supported by Nature Science Foundation of China (grant No. 81771899), Jiangsu Province Hospital of peak academic personnel training 
project (grant No. y2018rc04), and the Postgraduate Research \& Practice Innovation Program of Jiangsu Province (grant No. SJCX20_0514).

\section{Footnote}

Reporting Checklist: The authors have completed the TRIPOD reporting checklist. Available at https://dx.doi. org/10.21037/gs-21-38

Data Sharing Statement: Available at https://dx.doi. org/10.21037/gs-21-38

Conflicts of Interest: All authors have completed the ICMJE uniform disclosure form (available at https://dx.doi. org/10.21037/gs-21-38). The authors have no conflicts of interest to declare.

Ethical Statement: The authors are accountable for all aspects of the work in ensuring that questions related to the accuracy or integrity of any part of the work are appropriately investigated and resolved. The study was conducted in accordance with the Declaration of Helsinki (as revised in 2013). The study was approved by the institutional review board of the Affiliated Hospital of the Nanjing University of Chinese Medicine (No. 2016NL005-02: the registration number of ethics board) and individual consent for this retrospective analysis was waived.

Open Access Statement: This is an Open Access article distributed in accordance with the Creative Commons Attribution-NonCommercial-NoDerivs 4.0 International License (CC BY-NC-ND 4.0), which permits the noncommercial replication and distribution of the article with the strict proviso that no changes or edits are made and the original work is properly cited (including links to both the formal publication through the relevant DOI and the license). See: https://creativecommons.org/licenses/by-nc-nd/4.0/.

\section{References}

1. Siegel RL, Miller KD, Jemal A. Cancer statistics, 2020. CA Cancer J Clin 2020;70:7-30.

2. Koh WJ, Abu-Rustum NR, Bean S, et al. Uterine Neoplasms, Version 1.2018, NCCN Clinical Practice Guidelines in Oncology. J Natl Compr Canc Netw 2018;16:170-99.

3. Lavaud P, Fedida B, Canlorbe G, et al. Preoperative MR imaging for ESMO-ESGO-ESTRO classification of endometrial cancer. Diagn Interv Imaging 2018;99:387-96.

4. Lee SW, Lee TS, Hong DG, et al. Practice guidelines for management of uterine corpus cancer in Korea: a Korean Society of Gynecologic Oncology Consensus Statement. J Gynecol Oncol 2017;28:e12.

5. Liu J, Yuan F, Wang S, et al. The ability of ADC measurements in the assessment of patients with stage I endometrial carcinoma based on three risk categories. Acta Radiol 2019;60:120-8.

6. Lee JY, Kim YH, Lee JM, et al. Role of preoperative magnetic resonance imaging and histological assessment in identifying patients with a low risk of endometrial cancer: a Korean Gynecologic Oncology Group ancillary study. Oncotarget 2017;8:106009-16.

7. Al Nemer AM, Al Bayat MI, Al Qahtani NH. The accuracy of endometrial sampling for the diagnosis of patterns of endometrial pathology in women presenting with abnormal uterine bleeding. More conservative therapeutic approaches. Saudi Med J 2019;40:815-9.

8. Tanaka T, Terai Y, Ono YJ, et al. Preoperative MRI and intraoperative frozen section diagnosis of myometrial invasion in patients with endometrial cancer. Int J Gynecol Cancer 2015;25:879-83.

9. Luomaranta A, Butzow R, Pauna AR, et al. Combined use of endometrial sample and magnetic resonance imaging in the preoperative risk-stratification of endometrial carcinomas. Acta Obstet Gynecol Scand 2015;94:95-101.

10. Colombo N, Creutzberg C, Amant F, et al. ESMOESGO-ESTRO Consensus Conference on Endometrial Cancer: Diagnosis, Treatment and Follow-up. Int J Gynecol Cancer 2016;26:2-30.

11. Nougaret S, Reinhold C, Alsharif SS, et al. Endometrial Cancer: Combined MR Volumetry and Diffusionweighted Imaging for Assessment of Myometrial and Lymphovascular Invasion and Tumor Grade. Radiology 2015;276:797-808.

12. Chattopadhyay S, Cross P, Nayar A, et al. Tumor size: a better independent predictor of distant failure and death than depth of myometrial invasion in International Federation of Gynecology and Obstetrics stage I endometrioid endometrial cancer. Int J Gynecol Cancer 2013;23:690-7.

13. Zhang Q, Yu X, Lin M, et al. Multi-b-value diffusion weighted imaging for preoperative evaluation of risk stratification in early-stage endometrial cancer. Eur J Radiol 2019;119:108637. 
14. Shen Y, Lv F, Xiao Z, et al. Utility of the relative apparent diffusion coefficient for preoperative assessment of low risk endometrial carcinoma. Clin Imaging 2019;56:28-32.

15. Creasman WT, Morrow CP, Bundy BN, et al. Surgical pathologic spread patterns of endometrial cancer. A Gynecologic Oncology Group Study. Cancer 1987;60:2035-41.

16. Dane C, Bakir S. The effect of myometrial invasion on prognostic factors and survival analysis in endometrial carcinoma. Afr Health Sci 2019;19:3235-41.

17. Mhawech-Fauceglia P, Yan L, Liu S, et al. ER+/PR+ / TFF3+/IMP3-immunoprofile distinguishes endometrioid from serous and clear cell carcinomas of the endometrium: a study of 401 cases. Histopathology 2013;62:976-85.

18. Masjeed NMA, Khandeparkar SGS, Joshi AR, et al. Immunohistochemical Study of ER, PR, Ki67 and p53 in Endometrial Hyperplasias and Endometrial Carcinomas. J Clin Diagn Res 2017;11:EC31-EC34.

19. Guan J, Xie L, Luo X, et al. The prognostic significance of estrogen and progesterone receptors in grade I

Cite this article as: Chen J, Fan W, Gu H, Zhang W, Liu Y, Wang Y, Pan Z, Wang Z. Preoperative MRI and immunohistochemical examination for the prediction of highrisk endometrial cancer. Gland Surg 2021;10(7):2180-2191. doi: $10.21037 /$ gs-21-38 and II endometrioid endometrial adenocarcinoma: hormone receptors in risk stratification. J Gynecol Oncol 2019;30:e13.

20. Di Donato V, Iacobelli V, Schiavi MC, et al. Impact of Hormone Receptor Status and Ki-67 Expression on Disease-Free Survival in Patients Affected by High-risk Endometrial Cancer. Int J Gynecol Cancer 2018;28:505-13.

21. Han G, Sidhu D, Duggan MA, et al. Reproducibility of histological cell type in high-grade endometrial carcinoma. Mod Pathol 2013;26:1594-604.

22. Weinberger V, Bednarikova M, Hausnerova J, et al. A novel approach to preoperative risk stratification in endometrial cancer: the added value of immunohistochemical markers. Front Oncol 2019;9:265.

23. Yang B, Shan B, Xue X, et al. Predicting Lymph Node Metastasis in Endometrial Cancer Using Serum CA125 Combined with Immunohistochemical Markers PR and Ki67, and a Comparison with Other Prediction Models. PLoS One 2016;11:e0155145. 